\title{
Study on the AFM Force Curve Common Errors and Their Effects on the Calculated Nanomechanical Properties of Materials
}

\author{
D. Almasi, ${ }^{1,2}$ R. Sharifi, ${ }^{3}$ M. R. Abdul Kadir, ${ }^{2}$ G. Krishnamurithy, ${ }^{4}$ and T. Kamarul ${ }^{5}$ \\ ${ }^{1}$ Young Researchers and Elite Club, Kermanshah Branch, Islamic Azad University, Kermanshah, Iran \\ ${ }^{2}$ Medical Devices \& Technology Group (MEDITEG), Faculty of Bioscience and Medical Engineering, \\ Universiti Teknologi Malaysia, 81310 Skudai, Johor, Malaysia \\ ${ }^{3}$ Department of Endodontics, School of Dentistry, Kermanshah University of Medical Sciences, Kermanshah, Iran \\ ${ }^{4}$ Institute of Translational Medicine, University of Liverpool, Sherrington Building, Liverpool L69 3GE, UK \\ ${ }^{5}$ Department of Orthopaedic Surgery (NOCERAL), Faculty of Medicine, University of Malaya, 50603 Kuala Lumpur, Malaysia
}

Correspondence should be addressed to R. Sharifi; r.sharifi@kums.ac.ir

Received 12 August 2016; Accepted 29 September 2016

Academic Editor: Sheng-Rui Jian

Copyright ( $\odot 2016$ D. Almasi et al. This is an open access article distributed under the Creative Commons Attribution License, which permits unrestricted use, distribution, and reproduction in any medium, provided the original work is properly cited.

\begin{abstract}
The atomic force microscope (AFM) force curve has been widely used for determining the mechanical properties of materials due to its high resolution, whereby very low (piconewton) forces and distances as small as nanometers can be measured. However, sometimes the resultant force curve obtained from AFM is slightly different from those obtained from a more typical nanoindentation force curve due to the AFM piezo's hysteresis. In this study the nanomechanical properties of either a sulfonated polyether ether ketone (SPEEK) treated layer or bare polyether ether ketone (PEEK) were evaluated via AFM nanoindentation and a nanomechanical test system to probe the possible error of the calculated nanomechanical properties due to the AFM piezo's hysteresis. The results showed that AFM piezo's hysteresis caused the error in the calculated nanomechanical properties of the materials.
\end{abstract}

\section{Introduction}

Due to the increased applications of thin film polymeric materials and polymeric coating layers in different fields of research, probing the nanomechanical properties of polymeric materials has become essential to scientists. Based on Buckle's one-tenth rule for probing the mechanical properties of a coating or thin layer of a particular material, the maximum indentation depth must be less than one-tenth of the thickness of that layer to prevent an effect of the substrate in the resultant force curve [1]. High resolution equipment for measuring indentation force curves is therefore required. Atomic force microscopy (AFM) and nanomechanical test system have been used widely to probe the nanomechanical properties of the surface of materials.

The atomic force microscope was invented by Binning and Smith [2]. Burnham and Colton [3] used AFM in a nanoindentation study for the first time and measured nanomechanical properties of material surfaces at piconewton resolution. The AFM nanoindentation technique has since been used on various types of materials such as rat fibroblasts [4], tomato fruit cells [5], scaffolds [6], gels [7, 8], bulk metallic glass [9], polymers [10, 11], and especially thin coating layer [12]. However, there are some common errors in the AFM force curve due to the AFM piezo's hysteresis and using cantilevers with low spring constant which make it difficult or even impossible to extract the mechanical properties of the material from the AFM's force curve [13-18]. The nanomechanical test system (NTS) is another example of typical equipment that has been used to draw force-indentation depth graphs [19-21] and has been used to measure Young's modulus for various materials such as hydroxyapatite [22] and titanium [23]. Several formulations to calculate the elastic modulus from the force curve are 


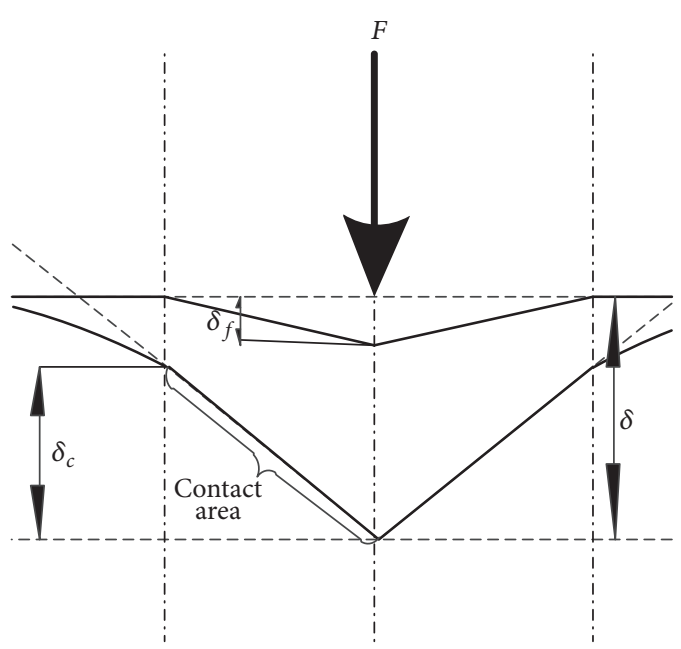

(a)

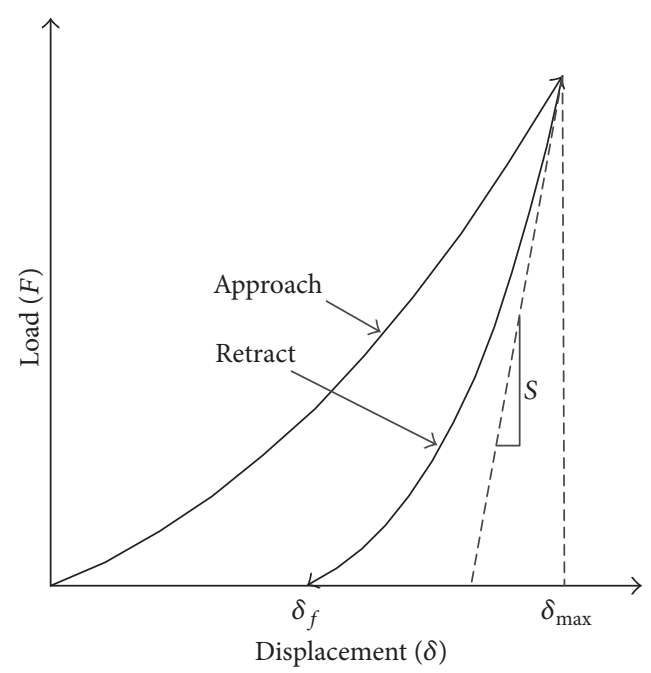

(b)

Figure 1: (a) Surface profile during loading with an indenter; (b) schematic of indentation force curve [28].

available, such as those by Johnson et al. (JKR) [24, 25], Derjaguin et al. (DMT) [26], Hertz [27], and Oliver and Pharr [28]. Each of the formulations has its unique strengths and limitations.

This comparative study was conducted to evaluate the effect of the AFM piezo hysteresis on the calculated mechanical properties of polymeric materials, compared with nanomechanical test system. For this reason Young's modulus of a treated layer of PEEK in sulfuric acid (i.e., SPEEK) was evaluated, as well as that of bare PEEK using AFM and nanomechanical test system. These materials were chosen as PEEK is relatively stiff and SPEEK is relatively soft in comparison with the cantilever's spring constant. Two formulations to calculate material properties from the AFM force curveHertz and Oliver-Pharr-were then used to analyze the two materials in terms of their suitability.

\section{Materials and Methods}

2.1. Sample Preparation. Twelve cylindrical samples of implant grade PEEK Optima ${ }^{\circledR}$ (Invibio) with a height of $0.8 \mathrm{~cm}$ and a diameter of $1.9 \mathrm{~cm}$ were used in the experiment. These samples were ground with 400-grit silicon carbide paper to reduce their surface roughness. After grinding, the samples were cleaned ultrasonically with acetone for 10 minutes [29]. For preparation of SPEEK samples, the ground PEEK disks were immersed in 95-97\% sulfuric acid for 5 minutes, followed by washing with deionized water several times until the $\mathrm{pH}$ of the water reached the neutral value of 7 [30].

\subsection{Numerical Model to Calculate Young's Modulus}

2.2.1. The Oliver-Pharr Model. The Oliver-Pharr model can be used to calculate Young's modulus of materials from an indentation force curve. This technique relies on the fact that the displacements recovered during unloading are largely elastic. In this case Young's modulus can be determined by elastic punch theory from the indentation force curve [28, 31]. Figure 1(a) shows the schematic of a surface profile during indentation, and Figure 1(b) shows the schematic of a force curve of a typical nanoindentation. The offset in Figure 1(b) is the residual plastic indentation depth $\left(\delta_{f}\right)$.

In nanoindentation studies of polymeric materials the elastic properties of the indenter can be ignored [32] due to the large differences in the elastic modulus between the tip and the sample. The following Oliver-Pharr equations [31] were based on this assumption. For a pyramid indenter with an angle $\alpha$, the projected contact area $\left(A_{c}\right)$ can be calculated from the contact depth $\left(\delta_{c}\right)$ via the following:

$$
A_{c}=4 \delta_{c}^{2} \tan \alpha
$$

And the contact depth $\left(\delta_{c}\right)$ can be obtained from (2) at peak load, where stiffness $(S)$ can be calculated by measuring the slope of the unloading part of the force curve at maximum indentation depth $\left(\delta_{\max }\right)$.

$$
\delta_{c}=\delta_{\max }-\varepsilon \frac{F_{\max }}{S},
$$

where $F_{\max }$ is the load at the depth of maximum indentation. The geometric constant of $\varepsilon$ is 0.72 for pyramidal and conical indenters [28]. Finally, to calculate Young's modulus $(E)$, the following can be used:

$$
E=\frac{S \sqrt{\pi}}{2 \sqrt{A_{c}}} .
$$

After unloading, the elastic deformation is completely recovered and only plastic indentation remains.

2.2.2. The Hertz Contact Model. The Hertz model can also be used to calculate Young's modulus of a material from 


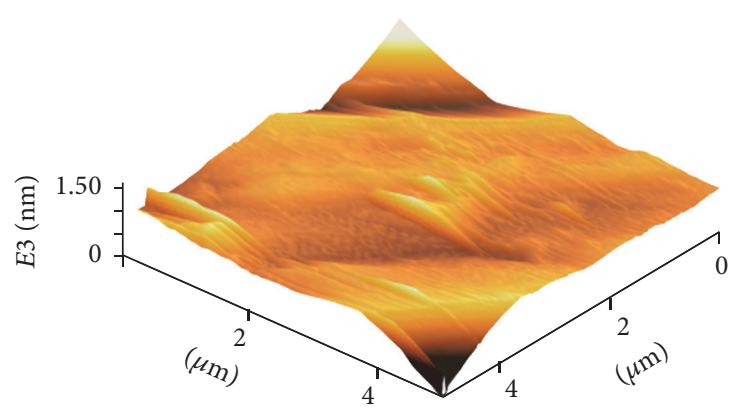

(a)

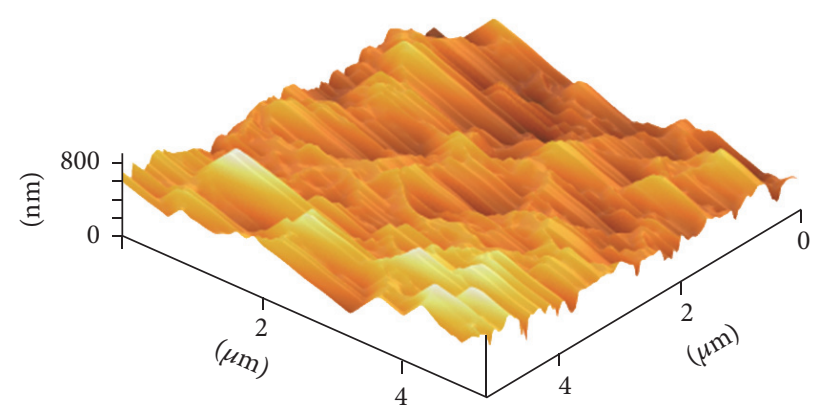

(b)

Figure 2: AFM 3D height image of (a) SPEEK and (b) PEEK.

its indentation force curve. Based on this model the sample is assumed to be linear in its elasticity and an isotropic solid, and the indenter is considered as a rigid material [27, 33]. Most studies have used the approach part of the force curve to calculate Young's modulus $[5,6,33]$; however, the average value of the approach-retract portion has also been considered [11]. For the four-sided pyramid indenter used in AFM, the Hertz model for calculating Young's modulus utilized the following [34]:

$$
F=\frac{E}{\left(1-v^{2}\right)} \frac{\tan \alpha}{\sqrt{2}} \delta^{2},
$$

where $\alpha$ is the face angle of the cantilever and $v$ is Poisson's ratio of the sample. For the Berkovich three-sided indenter used in the NTS, the Hertz model for calculating Young's modulus utilized the following $[33,35]$ :

$$
F=\frac{E}{\left(1-v^{2}\right)} \frac{2 * \tan \alpha}{\pi} \delta^{2}
$$

In this study Poisson's ratio of the SPEEK layer was assumed to be zero due to the high porosity of this layer, and Poisson's ratio of PEEK was assumed to be 0.4 [36].

2.3. AFM Nanoindentation. A Seiko SPA 300 atomic force microscope with a SPI 3700 probe station was used to evaluate the mechanical properties of the samples. An Olympus microcantilever (OMCL-TR400PSA-3) with a silicon nitride hollow pyramid indenter with a 70-degree indenter angle was used for the test. The cantilever's length was $100 \mu \mathrm{m}$ with a spring constant of $0.08 \mathrm{~N} / \mathrm{m}$. The AFM was run in force curve mode and a scan size of $20 \mu \mathrm{m} \times 20 \mu \mathrm{m}$. The force curve graph obtained from the test was used to calculate the elastic modulus. Three AFM nanoindentation tests were made per sample.

2.4. Nanomechanical Test System. The Hysitron TI 750D Ubi nanomechanical test system with the Berkovich indenter tip was used for the nanoindentation test. Three nanoindentation tests were made per sample. The maximum applied load was chosen based on a preliminary experiment considering the one-tenth indentation rule [1]. During the preliminary experiment, the applied load was varied from $200 \mu \mathrm{N}$ to $1000 \mu \mathrm{N}$, and the load-displacement graphs were analyzed to extract the maximum penetration depth, which must be less than one-tenth of the coating thickness. The results showed that $200 \mu \mathrm{N}$ was suitable. The chosen loading rate was $5 \mu \mathrm{N} / \mathrm{s}$ and the holding time was $300 \mathrm{~s}$.

\section{Results}

3.1. AFM and Nanomechanical Test System Results. Figure 2 shows the AFM 3D height image of the surface of PEEK and SPEEK. As can be seen the surface roughness of the PEEK increased after sulphonation due to the diffusion of the sulfuric acid [37]. Figure 3 shows the force-piezo displacement $\left(Z_{p}\right)$ for SPEEK and PEEK obtained from the AFM. Figure 3(a) shows the force-piezo displacement of SPEEK to show the effect of snap-in and snap-out, which occurs for hydrophilic materials. Figures 3(a) and 3(b) show the method of extracting the indentation depth from the force curve for SPEEK and PEEK. Figure 4 shows the force curves of the SPEEK and PEEK via the NTS.

3.2. Analysis of the AFM Results. The SPEEK AFM force curve showed the effect of snap-in and snap-out (Figure 3(a)). This phenomenon was not apparent for the PEEK AFM force curve (Figure 3(b)) and the NTS force curve results (Figure 4).

Another difference between AFM and NTS force curves which could happen is the reverse position of the approach and retract curve in the AFM's force curve. The retract portion of the NTS force curve is located on the right side of the approach portion (Figure 4). However, the force curve graph for the AFM shows the reverse (Figure 3 ). As a result, the residual indentation depth $\left(\delta_{f}\right)$ and contact area between the sample and indenter $\left(A_{c}\right)$ at maximum load cannot be extracted from the AFM force curve graph with reverse approach and retract part. Thus, Young's modulus and hardness of the material cannot be calculated via the Oliver and Pharr model. However, if an assumption is made that the residual indentation is zero, the retract portion of the AFM force curve [4] can be used to calculate the elastic modulus and hardness using the Oliver and Pharr model. This assumption, however, causes an inherent error 


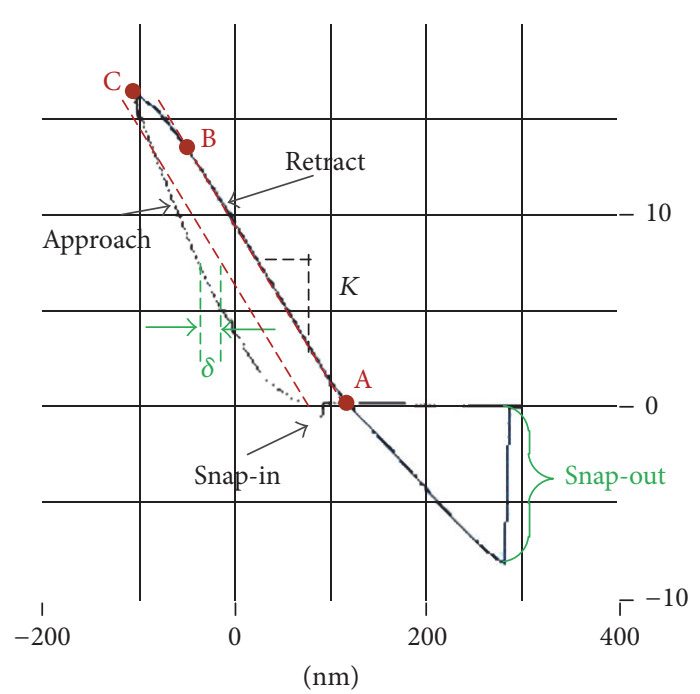

(a)

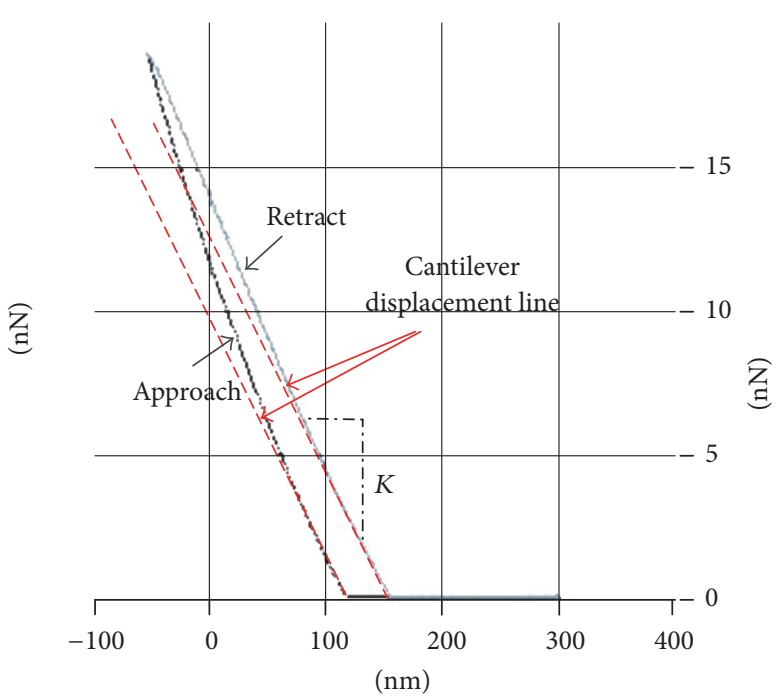

(b)

FIgURE 3: Force-piezo displacement for (a) SPEEK and (b) PEEK via AFM.

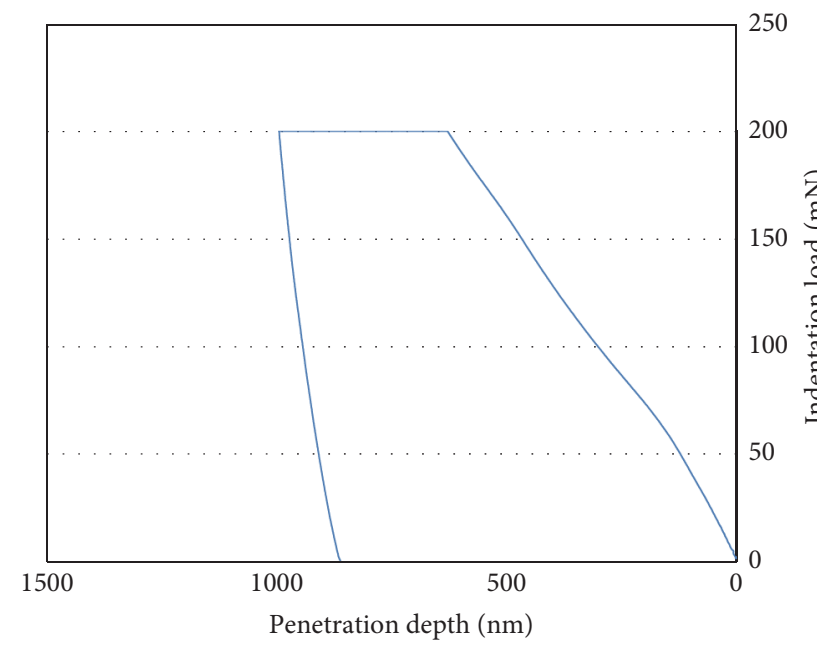

(a)

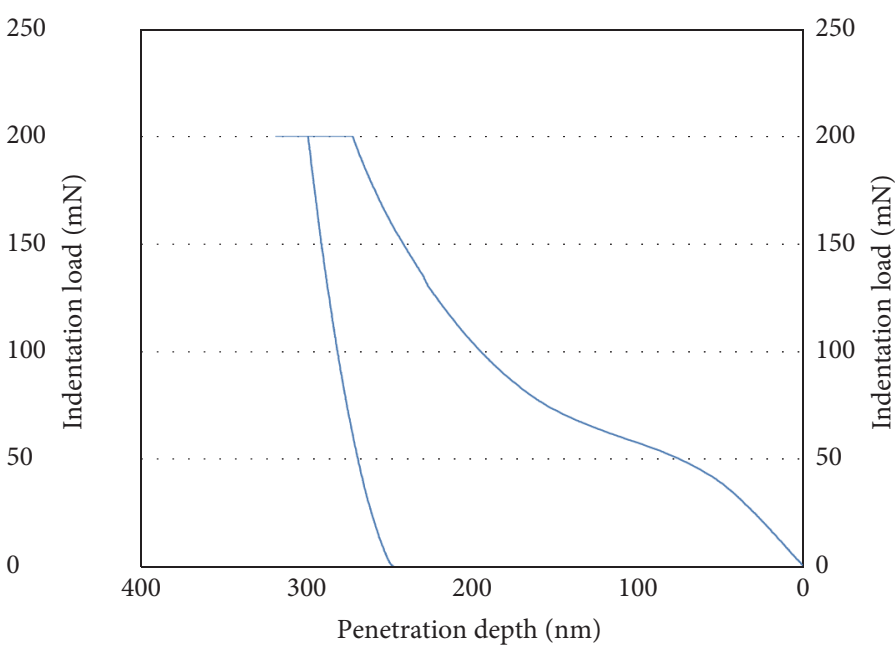

(b)

FIGURE 4: Force-indentation depth of (a) SPEEK and (b) PEEK via the NTS.

in the calculated Young's modulus and hardness. The reverse position of the approach-retract parts of the force curve is one of the biggest problems regarding the hysteresis of the AFM's piezo scanner and makes it difficult and sometimes impossible to analyze the AFM's force curve graph.

Another consideration that must be taken into account when analyzing AFM force curve is that the force versus piezo displacement $\left(Z_{p}\right)$ must be converted into force-indentation depth $(\delta)$ graph [39]. Figure 3 schematically shows the method of extracting the penetration $(\delta)$ from $F-Z_{p}$ graph. For calculating the indentation depth, a line with a slope value of $K$-the cantilever displacement line-must be drawn from the first approach point of the force curve. This line shows that the cantilever displaced with an assumption of fixed spring constant factor (Figure 3(b)). The indentation depth can be obtained from the piezo displacement via the following $[16,40]$ :

$$
\delta=Z_{p}-\frac{F}{K}
$$

As can be seen in Figure 3(a), the indentation $(\delta)$ for SPEEK is positive for the initial few nanometer piezo displacement in the approach part of the force curve. However, due to the nonlinearity of the cantilever spring properties and AFM piezo's hysteresis, the indentation became negative in the last few nanometer piezo displacement at the end of the approach part of the force curve. The negative value for indentation constitutes an error. Figure 5 shows the calculated 
TABLE 1: The calculated parameter based on the Oliver-Pharr model via the NTS.

\begin{tabular}{lcccccc}
\hline Sample & $\delta_{c}(\mathrm{~nm})$ & $S(\mu \mathrm{N} / \mathrm{nm})$ & $A_{c}\left(\mathrm{~nm}^{2}\right)$ & $\delta_{\max }(\mathrm{nm})$ & $\delta_{f}(\mathrm{~nm})$ & $E(\mathrm{MPa})$ \\
\hline SPEEK & $926 \pm 54$ & $2.16 \pm 0.24$ & $21649253 \pm 237384$ & $995 \pm 73$ & $838 \pm 62$ & $411 \pm 16$ \\
PEEK & $276 \pm 36$ & $6.51 \pm 0.18$ & $2143810 \pm 295375$ & $318 \pm 38$ & $241 \pm 46$ & $3937 \pm 149$ \\
\hline
\end{tabular}

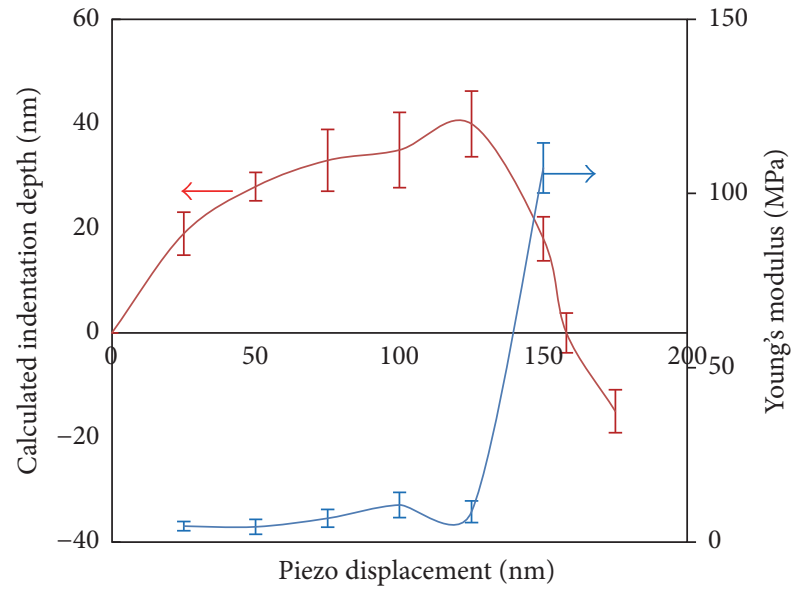

Calculated indentation depth (nm)
_ Young's modulus (Mpa)

Figure 5: Indentation depth versus piezo displacement of SPEEK using the approach portion of the force curve via AFM and calculated Young's modulus versus piezo displacement of SPEEK using the Hertz model.

indentation depth versus piezo displacement for SPEEK. For the first part of the indenter approach, the calculated indentation increased with increasing force, but as the load increases further, the calculated indentation decreased and eventually ended with a negative value, which is impossible. This happened due to the increase in error as the load increases. Nevertheless, the first part of the approach of the force curve of SPEEK (Figure 3(a)) can still be used for calculation using the Hertz model.

In Figure 3(a) the cantilever displacement line (A-B) overlaps the piezo displacement line, thus resulting in zero depth of indentation for the retract part of the AFM force curve of SPEEK. However, for region B-C there were minute differences resulting in a very small amount of indentation depth. Figure 6 shows the force-calculated indentation depth for the retract portion of the SPEEK's force curve. Since the tangent at maximum force does not cross the $x$-axis, that is, the indentation depth, the contact indentation depth cannot be obtained and therefore the elastic modulus cannot be calculated using this curve.

The calculated Young's modulus of the SPEEK layer by AFM nanoindentation via the Hertz model is shown in Figure 5. As can be seen, the calculated Young's modulus between $25 \mathrm{~nm}$ and $75 \mathrm{~nm}$ piezo displacement is $5.2 \pm 1.6 \mathrm{MPa}$, but with increasing piezo displacement the value increased to 107.3 MPa at $150 \mathrm{~nm}$. The elastic properties of SPEEK cannot

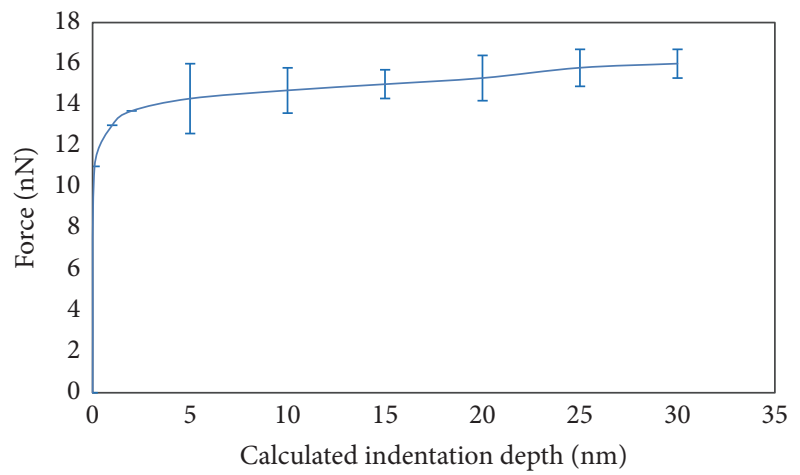

FIGURE 6: Force versus indentation depth of SPEEK from the retract portion of the force curve via AFM.

be calculated using the Oliver-Pharr model, as the contact area $\left(A_{c}\right)$ cannot be extracted from the force-indentation graph.

Figure 3(b) shows the force curve graph for PEEK. Compared to SPEEK, snap-in was not visible. The cantilever displacement line for the approach and retract portions overlapped with the piezo displacement line for the first few piezo displacement, resulting in zero indentation depth. However, with increasing of the value of the piezo displacement the force curve is located at the right side of the cantilever displacement line, which means a negative value for the indentation depth. The AFM indentation result for PEEK is different from SPEEK, showing that cantilever with low spring constant is not suitable for use in calculating the material properties of hard materials.

3.3. Analysis of the Nanomechanical Test System Results. Table 1 shows the calculated elasticity modulus of the SPEEK and PEEK based on the NTS force curve (Figure 4) via the Oliver-Pharr model.

Figure 7 shows the calculated modulus of elasticity of SPEEK and PEEK based on the Hertz model. As can be seen, the calculated elastic modulus was different for different indentation loads. Young's modulus for SPEEK with indentation loads between 150 and $200 \mu \mathrm{N}$ was $305 \pm$ $46 \mathrm{MPa}$, which was close to the calculated elastic modulus via the Oliver-Pharr method (411 MPa). The calculated elastic modulus of PEEK with indentation loads between 150 and $200 \mu \mathrm{N}$ was $1500 \pm 40 \mathrm{MPa}$, which was less than half of the calculated elastic modulus according to the Oliver-Pharr model $(3937 \mathrm{MPa})$. A summary of the calculated elastic modulus and the disadvantages of each type of equipment and numerical model are shown in Table 2. 


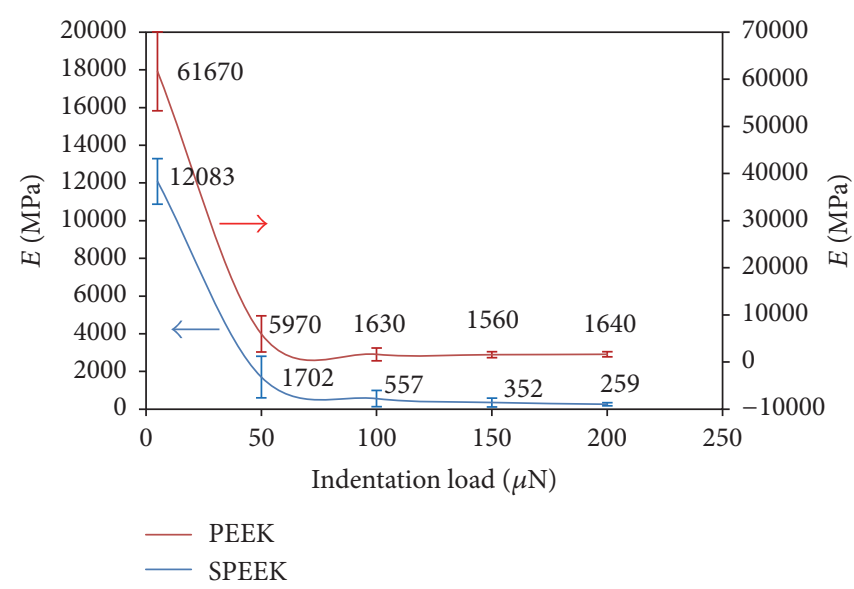

FIGURE 7: Calculated Young's modulus versus piezo displacement of (a) SPEEK and (b) PEEK using the Hertz model via the NTS.

TABLE 2: Summary of the calculated elastic modulus and the disadvantages of each type of equipment and numerical model used in this study.

\begin{tabular}{|c|c|c|c|c|c|}
\hline Materials & Equipment & Numerical model & Assumptions & Disadvantages & Calculated $E(\mathrm{MPa})$ \\
\hline \multirow{4}{*}{ PEEK } & \multirow{2}{*}{ AFM } & Oliver-Pharr & - & The indentation depth could not & - \\
\hline & & Hertz & $v=0$ & be extracted from the force-piezo & - \\
\hline & \multirow{2}{*}{$\begin{array}{l}\text { Nanomechanical test } \\
\text { system }\end{array}$} & Oliver-Pharr & - & - & $3937 \pm 149$ \\
\hline & & Hertz & $v=0.4$ & $\begin{array}{l}\text { The indentation depth has too } \\
\text { much error [38] }\end{array}$ & $1600 \pm 40$ \\
\hline \multirow{4}{*}{ SPEEK } & \multirow[t]{2}{*}{ AFM } & Oliver-Pharr & $\delta_{f}=0$ & $\begin{array}{l}\text { The contact indentation depth } \\
\text { cannot be extracted }\end{array}$ & - \\
\hline & & Hertz & $v=0$ & $\begin{array}{l}\text { The indentation depth can only } \\
\text { be extracted for the first few } \\
\text { nanometers }\end{array}$ & $5.6 \pm 1.1$ \\
\hline & \multirow{2}{*}{$\begin{array}{l}\text { Nanomechanical test } \\
\text { system }\end{array}$} & Oliver-Pharr & - & - & $411 \pm 16$ \\
\hline & & Hertz & $v=0.4$ & - & $510 \pm 250$ \\
\hline
\end{tabular}

\section{Discussion}

We have compared the outcome of the AFM force curve and nanomechanical test force curve for two types of polymeric materials-PEEK and SPEEK. The AFM force curve for SPEEK shows snap-in and snap-out due to the hydrophilicity of the material. However, this does not occur in PEEK which is hydrophobic $[6,39,41]$. The observed differences between the two materials were not apparent if the NTS was used, as its indenter was not linked to a cantilever similar to that found in the AFM. Snap-in is a condition where the cantilever suddenly bends or is attracted to the sample due to the van der Waals attraction [42]. After contact, the indenter will bend upwards as it is continually pushed by the sample in the upwards direction, thus increasing the contact force on the sample. During retraction, the indenter is initially prevented from separation due to adhesive forces which includes the van der Waals forces, the electrostatic force, capillary force, and the chemical bonds $[42,43]$. At a certain distance of retraction, the indenter suddenly snaps out as the adhesive forces can no longer sustain the separation load.

Another error in the AFM force curves which affects the prediction of mechanical properties is the reverse position of the approach and retract curves. This is a common error due to the hysteresis of the AFM's piezo scanner which has been reported by several researchers [13-18]. This error makes it impossible to extract the residual indentation depth $\left(\delta_{f}\right)$ and contact area between the sample and indenter. Even with the reverse portion of the approach and retract part, the elastic modulus can still be extracted from the force curve using the Hertz model [27, 33]. Also the Oliver-Pharr model can be used for calculating the elastic modulus with an assumption of zero residual indentation depth $\left(\delta_{f}\right)$.

In this particular comparative study, we found that the prediction of elastic modulus from the AFM was less accurate than the NTS due to the cantilever effect and piezo hysteresis from the AFM. For the NTS, the Oliver-Pharr model gave a more accurate prediction of the elastic modulus compared to the Hertz model [38].

\section{Conclusion}

A study was done to compare the predicted elastic modulus from two types of equipment-AFM and NTS. Force curves from both types of equipment were used with the 
mathematical models of Oliver-Pharr and Hertz to calculate Young's modulus of PEEK and SPEEK. We observed the following difficulties when analyzing mechanical properties via the AFM's force curve which are common in terms of using AFM for nanoindentation purpose. Firstly the force curve graph of PEEK could not be extracted from the force-piezo displacement due to the relatively high stiffness of PEEK compared to the cantilever's spring constant. Therefore using cantilever with higher cantilever spring constant is preferred for indentation purpose. Secondly the approach and retract portion of the AFM's force curve was reversed in comparison with the NTS force curve graph. The reversed curve was due to hysteresis of the piezo of the AFM equipment making it impossible to extract $\delta_{f}$ from the AFM force curve graph. In contrast, the NTS with the Oliver-Pharr model predicted Young's modulus of both PEEK and SPEEK accurately.

\section{Competing Interests}

The authors declare that there are no competing interests regarding the publication of this paper.

\section{References}

[1] I. Manika and J. Maniks, "Effect of substrate hardness and film structure on indentation depth criteria for film hardness testing," Journal of Physics D: Applied Physics, vol. 41, no. 7, Article ID 074010, 2008.

[2] G. Binnig and D. P. E. Smith, "Single-tube three-dimensional scanner for scanning tunneling microscopy," Review of Scientific Instruments, vol. 57, no. 8, pp. 1688-1689, 1986.

[3] N. A. Burnham and R. J. Colton, "Measuring the nanomechanical properties and surface forces of materials using an atomic force microscope," Journal of Vacuum Science \& Technology A, vol. 7, no. 4, pp. 2906-2913, 1989.

[4] M. Plodinec, M. Loparic, R. Suetterlin, H. Herrmann, U. Aebi, and C.-A. Schoenenberger, "The nanomechanical properties of rat fibroblasts are modulated by interfering with the vimentin intermediate filament system," Journal of Structural Biology, vol. 174, no. 3, pp. 476-484, 2011.

[5] A. Zdunek and A. Kurenda, "Determination of the elastic properties of tomato fruit cells with an atomic force microscope," Sensors, vol. 13, no. 9, pp. 12175-12191, 2013.

[6] Y. Zhu, Z. Dong, U. C. Wejinya, S. Jin, and K. Ye, "Determination of mechanical properties of soft tissue scaffolds by atomic force microscopy nanoindentation," Journal of Biomechanics, vol. 44, no. 13, pp. 2356-2361, 2011.

[7] E. K. Dimitriadis, F. Horkay, J. Maresca, B. Kachar, and R. S. Chadwick, "Determination of elastic moduli of thin layers of soft material using the atomic force microscope," Biophysical Journal, vol. 82, no. 5, pp. 2798-2810, 2002.

[8] C. D. Markert, X. Guo, A. Skardal et al., "Characterizing the micro-scale elastic modulus of hydrogels for use in regenerative medicine," Journal of the Mechanical Behavior of Biomedical Materials, vol. 27, pp. 115-127, 2013.

[9] W. Liang, Z. Ning, Z. Dang, and L. Wu, "Plastic deformation behaviors of Ni- and Zr-based bulk metallic glasses subjected to nanoindentation," Materials Characterization, vol. 86, pp. 290295, 2013.
[10] D. Tranchida, S. Piccarolo, and M. Soliman, "Nanoscale mechanical characterization of polymers by AFM nanoindentations: criticalr approach to the elastic characterization," Macromolecules, vol. 39, no. 13, pp. 4547-4556, 2006.

[11] J. Domke and M. Radmacher, "Measuring the elastic properties of thin polymer films with the atomic force microscope," Langmuir, vol. 14, no. 12, pp. 3320-3325, 1998.

[12] M. Sebastiani, A. Cusmà, E. Bemporad, and F. Carassiti, "Elastic anisotropy of coatings by AFM analysis of microindentations," Surface Engineering, vol. 30, no. 1, pp. 41-47, 2014.

[13] J. Z. Ai, X. P. Guo, and Z. Y. Chen, “The adsorption behavior and corrosion inhibition mechanism of anionic inhibitor on galvanic electrode in $1 \% \mathrm{NaCl}$ solution," Applied Surface Science, vol. 253, no. 2, pp. 683-688, 2006.

[14] Y. Chen, G. Zeng, S. S. Chen, Q. Feng, and Z. W. Chen, "AFM force measurements of the gp120-sCD4 and gp120 or CD4 antigen-antibody interactions," Biochemical and Biophysical Research Communications, vol. 407, no. 2, pp. 301-306, 2011.

[15] V. M. Masterson and X. Cao, "Evaluating particle hardness of pharmaceutical solids using AFM nanoindentation," International Journal of Pharmaceutics, vol. 362, no. 1-2, pp. 163-171, 2008.

[16] Influence of Soft Segment Content and Chain Length on the Physical Properties of Poly(ether ester) Elastomers and Fabrication of Honeycomb Pattern and Electrospun Fiber, Reactive and Functional Polymers, 2013.

[17] JPK Instruments AG, "A practical guide to AFM force spectroscopy and data analysis," Technical Note, JPK Instruments AG, Berlin, Germany, 2014.

[18] Q. Ouyang, K. Ishida, and K. Okada, "Investigation of microadhesion by atomic force microscopy," Applied Surface Science, vol. 169-170, pp. 644-648, 2001.

[19] C. A. Schuh, "Nanoindentation studies of materials," Materials Today, vol. 9, no. 5, pp. 32-40, 2006.

[20] O. Şahin, O. Uzun, U. Kölemen, and N. Uçar, "Mechanical characterization for $\beta$-Sn single crystals using nanoindentation tests," Materials Characterization, vol. 59, no. 4, pp. 427-434, 2008.

[21] D. J. Shuman, A. L. M. Costa, and M. S. Andrade, "Calculating the elastic modulus from nanoindentation and microindentation reload curves," Materials Characterization, vol. 58, no. 4, pp. 380-389, 2007.

[22] A. Dey, A. K. Mukhopadhyay, S. Gangadharan, M. K. Sinha, D. Basu, and N. R. Bandyopadhyay, "Nanoindentation study of microplasma sprayed hydroxyapatite coating," Ceramics International, vol. 35, no. 6, pp. 2295-2304, 2009.

[23] F. K. Mante, G. R. Baran, and B. Lucas, "Nanoindentation studies of titanium single crystals," Biomaterials, vol. 20, no. 11, pp. 1051-1055, 1999.

[24] K. L. Johnson, K. Kendall, and A. D. Roberts, "Surface energy and the contact of elastic solids," Proceedings of the Royal Society A: Mathematical, Physical and Engineering Sciences, vol. 324, no. 1558, pp. 301-313, 1971.

[25] D. Maugis and M. Barquins, "Adhesive contact of sectionally smooth-ended punches on elastic half-spaces: theory and experiment," Journal of Physics D: Applied Physics, vol. 16, no. 10, pp. 1843-1874, 1983.

[26] B. V. Derjaguin, V. M. Muller, and Y. P. Toporov, "Effect of contact deformations on the adhesion of particles," Journal of Colloid and Interface Science, vol. 53, no. 2, pp. 314-326, 1975. 
[27] H. R. Hertz, Ueber die Beruehrung Elastischer Koerper (On Contact Between Elastic Bodies), 1895.

[28] W. C. Oliver and G. M. Pharr, "Improved technique for determining hardness and elastic modulus using load and displacement sensing indentation experiments," Journal of Materials Research, vol. 7, no. 6, pp. 1564-1580, 1992.

[29] A. Rabiei and S. Sandukas, "Processing and evaluation of bioactive coatings on polymeric implants," Journal of Biomedical Materials Research Part A, vol. 101, no. 9, pp. 2621-2629, 2013.

[30] K. Janaki, S. Elamathi, and D. Sangeetha, "Development and characterization of polymer ceramic composites for orthopedic applications," Trends in Biomaterials \& Artificial Organs, vol. 22, no. 3, pp. 169-178, 2008.

[31] G. M. Pharr, W. C. Oliver, and F. R. Brotzen, "On the generality of the relationship among contact stiffness, contact area, and elastic modulus during indentation," Journal of Materials Research, vol. 7, no. 3, pp. 613-617, 1992.

[32] C. A. Clifford and M. P. Seah, "Quantification issues in the identification of nanoscale regions of homopolymers using modulus measurement via AFM nanoindentation," Applied Surface Science, vol. 252, no. 5, pp. 1915-1933, 2005.

[33] O. Olubi, D. Gadi, B. Sannigrahi, M. Williams, B. Baird, and I. Khan, "Fabrication of electroactive composite nanofibers of functionalized polymer and CNT capable of specifically binding with the IgE (Immunoglobulin E) antibody," Surface and Interface Analysis, vol. 46, no. 4, pp. 237-242, 2014.

[34] N. Gavara and R. S. Chadwick, "Determination of the elastic moduli of thin samples and adherent cells using conical atomic force microscope tips," Nature Nanotechnology, vol. 7, no. 11, pp. 733-736, 2012.

[35] A. C. Fischer-Cripps, "Review of analysis methods for submicron indentation testing," Vacuum, vol. 58, no. 4, pp.569-585, 2000.

[36] Victrex ${ }^{\circledR}$ PEEK 450G General Purpose, 2014, http://www.matweb.com/.

[37] D. Almasi, S. Izman, M. Assadian, M. Ghanbari, and M. R. Abdul Kadir, "Crystalline ha coating on peek via chemical deposition," Applied Surface Science, vol. 314, pp. 1034-1040, 2014.

[38] Introducing ortho PEEK, 2014.

[39] H.-J. Butt, B. Cappella, and M. Kappl, "Force measurements with the atomic force microscope: technique, interpretation and applications," Surface Science Reports, vol. 59, no. 1-6, pp. 1-152, 2005.

[40] B. Cappella and G. Dietler, Force Distance Curves by Atomic Force Microscopy, North-Holland, Amsterdam, The Netherlands, 1999.

[41] Y. Kaibara, K. Sugata, M. Tachiki, H. Umezawa, and H. Kawarada, "Control wettability of the hydrogen-terminated diamond surface and the oxidized diamond surface using an atomic force microscope," Diamond and Related Materials, vol. 12, no. 3-7, pp. 560-564, 2003.

[42] S. Maghsoudy-Louyeh and B. R. Tittmann, "Assessment of hydrous affinity of selected material," Colloids and Surfaces A: Physicochemical and Engineering Aspects, vol. 331, no. 3, pp. 268$274,2008$.

[43] Q. Ouyang, K. Ishida, and K. Okada, "Investigation of microadhesion by atomic force microscopy," Applied Surface Science, vol. 169-170, pp. 644-648, 2001. 


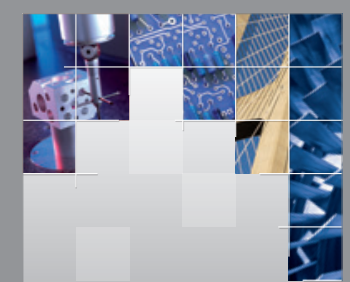

\section{Enfincering}
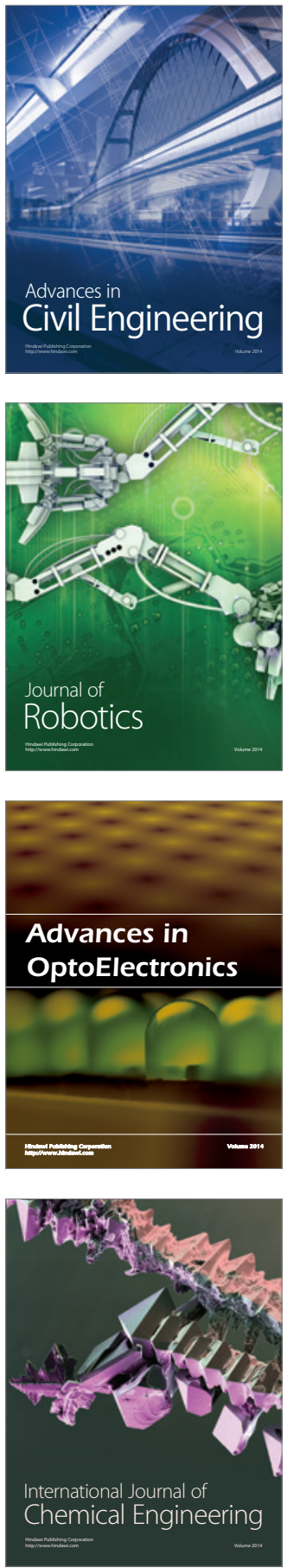

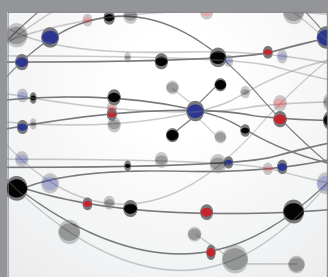

The Scientific World Journal

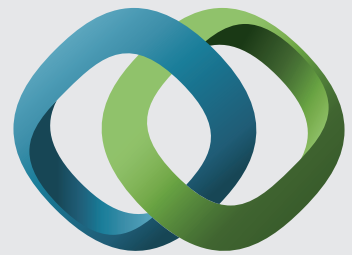

\section{Hindawi}

Submit your manuscripts at

http://www.hindawi.com
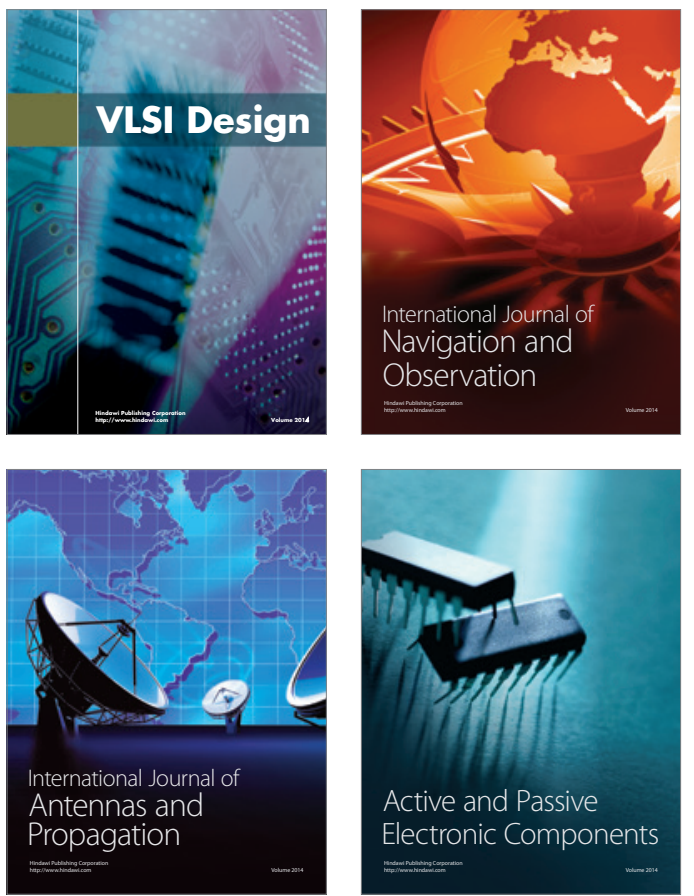
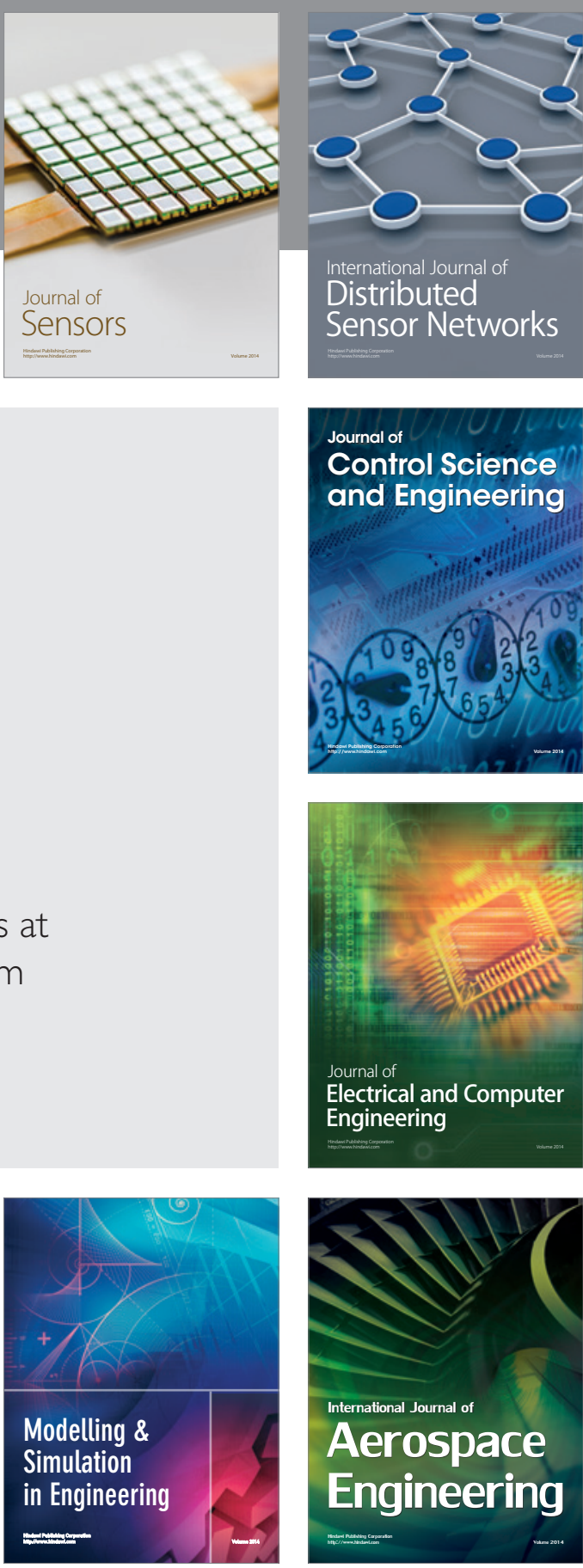

International Journal of

Distributed

Sensor Networks

Journal of

Control Science

and Engineering
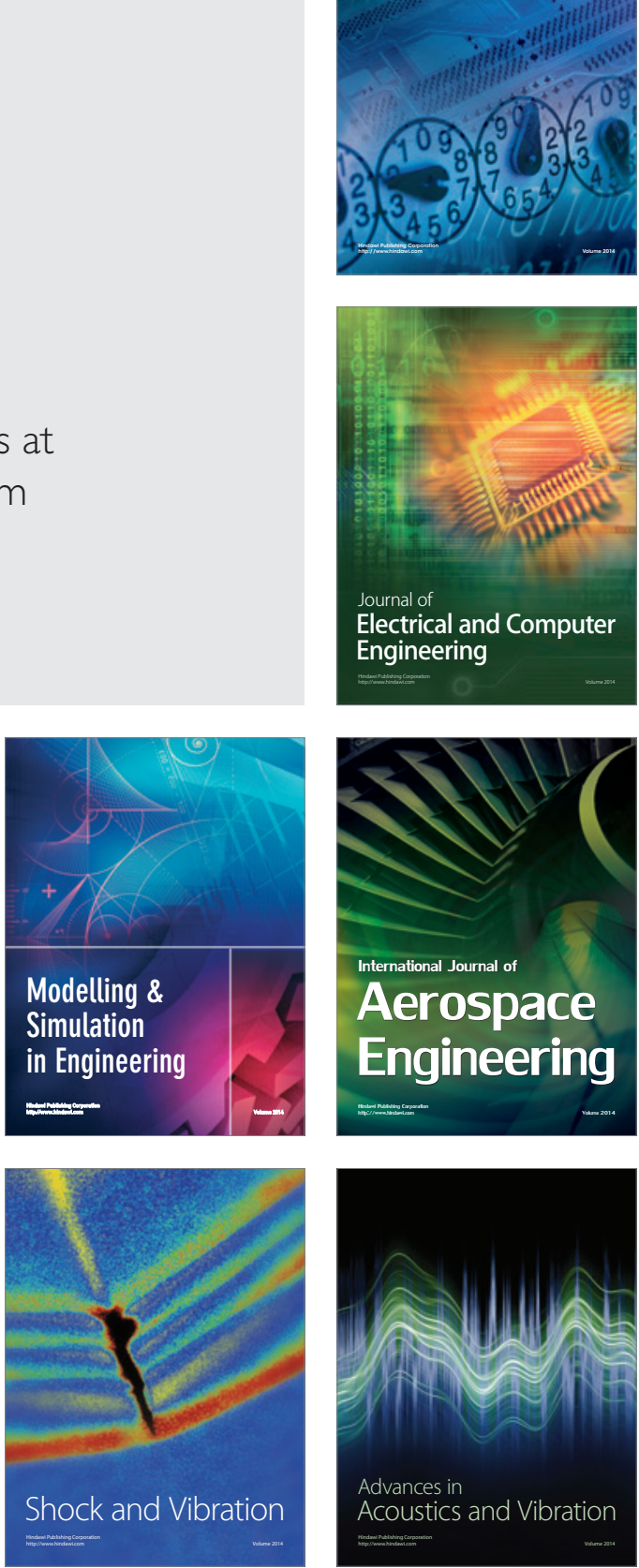